\title{
Whole-body transcriptome of selectively bred, resistant-, control-, and susceptible-line rainbow trout following experimental challenge with Flavobacterium psychrophilum
}

\author{
David Marancik', Guangtu Gao ${ }^{1}$, Bam Paneru' ${ }^{2}$, Hao Ma $^{3}$, Alvaro G. Hernandez ${ }^{4}$, Mohamed Salem², \\ Jianbo Yao ${ }^{3}$, Yniv Palti ${ }^{1}$ and Gregory D. Wiens ${ }^{1 *}$ \\ ${ }^{1}$ National Center for Cool and Cold Water Aquaculture, Agricultural Research Service, United States Department of Agriculture, Kearneysville, WV, USA \\ ${ }^{2}$ Department of Biology, Middle Tennessee State University, Murfreesboro, TN, USA \\ ${ }^{3}$ Animal and Nutritional Sciences, West Virginia University, Morgantown, WV, USA \\ ${ }^{4}$ High-Throughput Sequencing and Genotyping Unit, Roy J. Carver Biotechnology Center, University of Illinois at Urbana-Champaign, Urbana, IL, USA
}

\section{Edited by:}

Scott Newman, Genus, plc, USA

Reviewed by:

Hugo Murua Escobar, Medical

Faculty University of Rostock,

Germany

Dan Nonneman, Agricultural

Research Service, United States

Department of Agriculture, USA

\section{${ }^{*}$ Correspondence:}

Gregory D. Wiens, National Center

for Cool and Cold Water

Aquaculture, Agricultural Research

Service, United States Department of Agriculture, 11861 Leetown Rd.

Kearnyesville, WV 25430, USA

e-mail: greg.wiens@ars.usda.gov
Genetic improvement for enhanced disease resistance in fish is an increasingly utilized approach to mitigate endemic infectious disease in aquaculture. In domesticated salmonid populations, large phenotypic variation in disease resistance has been identified but the genetic basis for altered responsiveness remains unclear. We previously reported three generations of selection and phenotypic validation of a bacterial cold water disease (BCWD) resistant line of rainbow trout, designated ARS-Fp-R. This line has higher survival after infection by either standardized laboratory challenge or natural challenge as compared to two reference lines, designated ARS-Fp-C (control) and ARS-Fp-S (susceptible). In this study, we utilized $1.1 \mathrm{~g}$ fry from the three genetic lines and performed RNA-seq to measure transcript abundance from the whole body of naive and Flavobacterium psychrophilum infected fish at day 1 (early time-point) and at day 5 post-challenge (onset of mortality). Sequences from 24 libraries were mapped onto the rainbow trout genome reference transcriptome of 46,585 predicted protein coding mRNAs that included 2633 putative immune-relevant gene transcripts. A total of 1884 genes $(4.0 \%$ genome) exhibited differential transcript abundance between infected and mock-challenged fish (FDR $<0.05$ ) that included chemokines, complement components, tnf receptor superfamily members, interleukins, nod-like receptor family members, and genes involved in metabolism and wound healing. The largest number of differentially expressed genes occurred on day 5 post-infection between naive and challenged ARS-Fp-S line fish correlating with high bacterial load. After excluding the effect of infection, we identified 21 differentially expressed genes between the three genetic lines. In summary, these data indicate global transcriptome differences between genetic lines of naive animals as well as differentially regulated transcriptional responses to infection.

Keywords: Flavobacterium psychrophilum, bacterial cold water disease, selective breeding, disease resistance, aquaculture, immune gene, tnfrsf, rainbow trout genome

\section{INTRODUCTION}

Selective breeding programs contribute to increased aquaculture production through the generation of animals with improved resistance/tolerance toward infectious disease causing microorganisms (Gjedrem, 1983, 2005; Van Muiswinkel et al., 1999; Cock et al., 2009; Gjedrem et al., 2012). In 2005, a family-based selective breeding program was initiated at the National Center for Cool and Cold Water Aquaculture (NCCCWA) to improve rainbow trout (Oncorhynchus mykiss) survival following exposure to Flavobacterium psychrophilum (Silverstein et al., 2009). This pathogen is the etiologic agent of bacterial cold water disease (BCWD) and rainbow trout fry syndrome (RTFS), and causes considerable losses to the rainbow trout aquaculture industry within the U.S. and to trout and salmon populations worldwide (Nematollahi et al., 2003; Barnes and Brown, 2011). Infection of rainbow trout with $F$. psychrophilum typically results in mortality, ranging from 2 to $30 \%$ of the population, with higher losses caused by co-infection with the infectious hematopoietic virus. A further impact of the disease is that survival following infection has been associated with skeletal deformities (Kent et al., 1989; Madsen et al., 2001). Disease prevention is difficult as the pathogen is geographically wide spread, limited chemotherapeutants are available for treatment, and there is currently no commercial vaccine available in the U.S, although killed, subunit, and live-attenuated vaccines are all active areas of research (Gómez et al., 2014; Sundell et al., 2014). 
A pedigreed line of rainbow trout, designated ARS-Fp-R, has been subjected to over three generations of selection and demonstrates increased survival following experimental injection challenge (Hadidi et al., 2008; Silverstein et al., 2009; Leeds et al., 2010) and natural exposure (Wiens et al., 2013a), relative to a disease susceptible line, ARS-Fp-S, and a randomly bred control line, ARS-Fp-C. Current research goals at the NCCCWA includes elucidating intrinsic factors associated with survival of the ARSFp-R line to better understand mechanisms of how selection has altered the genetic control of disease resistance. Phenotypic studies have thus far shown that ARS-Fp-R line fish have decreased organ damage as determined by histopathology (Marancik et al., 2014 b) and fewer pathophysiologic changes in plasma biochemistry (Marancik et al., 2014a) during the acute-phase of disease following experimental challenge. Experiments that quantified splenic F. psychrophilum numbers on days 5 (Hadidi et al., 2008) and 9 post-infection (Marancik et al., 2014a) demonstrate significantly lower splenic bacterial loads in ARS-Fp-R line fish. It is likely these observed differences are a result of a differential immune response to infection.

Changes associated with host immunologic response can be elucidated by profiling alterations in host mRNA abundance between pathogen naive and infected animals (Martin et al., 2006; Beck et al., 2012; Langevin et al., 2012; Peatman et al., 2013; Pereiro et al., 2014; Shi et al., 2014). Previous studies of rainbow trout infected with F. psychrophilum demonstrate significant upregulation and downregulation of rainbow trout immune-relevant genes in the limited number of tissues examined (Overturf and LaPatra, 2006; Villarroel et al., 2008, 2009; Evenhuis and Cleveland, 2012; Langevin et al., 2012; Orieux et al., 2013; Henriksen et al., 2014). Microarray analysis of head kidney tissue from susceptible compared to resistant double-haploid rainbow trout lines, identified differences in basal gene expression as well as induction of antimicrobial peptides, complement, matrix metalloproteases, and chemokines 5 days post-infection (Langevin et al., 2012). Taken together, these studies suggest that the rainbow trout immune response to F. psychrophilum is likely multifactorial involving both innate and adaptive components.

In this manuscript, we quantify changes in gene transcript abundance between genetic lines with the following goals: (1) identify differentially regulated genes common to the host response to F. psychrophilum infection, (2) identify genes differentially regulated between lines in response to F. psychrophilum infection, and finally, (3) examine baseline differences in gene expression between naive animals that might contribute to the post-challenge phenotype. For this study, we utilized a comprehensive RNA-seq, transcriptome approach, starting with whole-body lysates from $1.1 \mathrm{~g}$ fry from pooled fish of naive or experimentally infected genetic lines. Experimental infection during the fry/juvenile lifestage allowed gene transcript profiling at a time when genetic lines express robust survival differences, and when epizootics and mortality are described as most severe in production environments (Branson, 1995; Decostere et al., 2000). Sequence reads were aligned to the recently released rainbow trout genome transcriptome (Berthelot et al., 2014) to which we added automated annotation and manual immune gene curation. In summary, we described common, whole-body gene transcriptional responses to early F. psychrophilum infection and potential differences associated with the innate response between genetic lines, and finally, compare our results with published gene expression studies utilizing F. psychrophilum challenged rainbow trout.

\section{MATERIALS AND METHODS ETHIC STATEMENT}

Fish were maintained at the NCCCWA and animal procedures were performed under the guidelines of NCCCWA Institutional Animal Care and Use Committee Protocols \#053 and \#076.

\section{EXPERIMENTAL ANIMALS}

The ARS-Fp-R, ARS-Fp-C, and ARS-Fp-S genetic lines were derived from the same base population, and thus differed only as a result of artificial selection for BCWD post-challenge survival (Wiens et al., 2013a). Single-sire $\times$ single-dam matings were made within genetic lines between 3-year-old females and 1-yearold neo-males as previously described (Marancik et al., 2014b). Water temperatures in the egg incubation jars were manipulated so that all families hatched within a 1-week period (Leeds et al., 2010). Eggs were pooled within-line at the eyed stage and reared in $\sim 12.5^{\circ} \mathrm{C}$ flow-through spring water. The ARS-Fp-R egg pool consisted of contributions from 43 full-sib families, the ARS-Fp-C egg pool consisted of 10 full-sib families, and the ARS-Fp-S egg pool consisted of 11 full-sib families. The resistant line eggs were progeny of dams that had undergone three generations of BCWD selection while the sires had undergone four generations of selection. The control-line eggs were progeny of parents that had undergone one generation of selection for increased resistance (2007 year class) and since that time, randomly bred. The susceptible-line eggs were progeny of parents that had undergone one generation of selection for increased susceptibility (2007 year class) and since that time, randomly bred (Wiens et al., 2013a). Eggs were pooled from a larger number of resistant line families as more resistant families are generated within the breeding program to apply selection differential, and thus this sampling design more accurately captured the genetic diversity within the resistant line. In addition, the resistant-line egg pool was part of a germplasm release and was utilized in additional challenge studies that will be reported elsewhere (Wiens, unpublished data).

All brood-stock and fish used in this study were certified to be free of common salmonid bacterial and viral pathogens by two independent diagnostic laboratories as described previously, and were negative for F. psychrophilum infection (Leeds et al., 2010; Wiens et al., 2013a). Prior to challenge, fry were allowed 1 week to acclimatize to challenge tanks. Mean body weight of the ARSFp-R line fish was $1.11 \pm 0.05 \mathrm{~g}$, the ARS-Fp-C line was $1.12 \pm$ $0.03 \mathrm{~g}$, and the ARS-Fp-S line was $0.98 \pm 0.04 \mathrm{~g}( \pm 1 \mathrm{SD}$, pooled weights of $n=4$ tanks) at the initiation of the challenge. In the challenge facility, photoperiod was adjusted weekly to maintain a natural lighting cycle, and at the time of RNA-seq sample collection, was $14.5 \mathrm{~h}$ light: $9.5 \mathrm{~h}$ dark. Water quality parameters have been described previously (Wiens et al., 2013a).

\section{RNA-seq EXPERIMENTAL DESIGN}

Bacterial challenge was carried out in the NCCCWA challenge facility with F. psychrophilum strain CSF-259-93 (initially 
provided by Dr. S. LaPatra, Clear Springs Foods, Inc.). This strain was previously isolated from a BCWD field-case and maintained at $-80^{\circ} \mathrm{C}$ in TYES media supplemented with $10 \%(\mathrm{v} / \mathrm{v})$ glycerol and has been consistently utilized as the challenge strain within the selective breeding program (Hadidi et al., 2008; Silverstein et al., 2009; Leeds et al., 2010; Wiens et al., 2013a) and the complete genome sequence determined (Wiens et al., 2014). Frozen stock was cultivated on TYES media for 5 days at $15^{\circ} \mathrm{C}$, suspended in PBS and O.D.525 adjusted to 0.4. Colony plate counts were performed in triplicate and recorded after 5 days incubation to estimate the challenge dose enumerated as viable CFU fish ${ }^{-1}$.

Each tank held fifty, randomly assigned fish and were supplied with $2.4 \mathrm{~L} \mathrm{~min}^{-1}$ of $12.5 \pm 0.1^{\circ} \mathrm{C}$ flow-through spring water. For each genetic line, two tanks of fish were challenged by F. psychrophilum injection and two tanks of fish were challenged by PBS injection and served as non-infected control animals. In total, 100 fish per line were anesthetized with $100 \mathrm{mg} / \mathrm{L}$ tricaine methanesulfonate (Tricaine-S, Western Chemical, Inc., Ferndale, WA) and intraperitoneally (IP) injected with $4.2 \times 10^{6} \mathrm{CFU}_{\text {fish }}{ }^{-1} \mathrm{~F}$. psychrophilum suspended in $10 \mu \mathrm{L}$ of chilled PBS or $10 \mu \mathrm{L}$ of chilled PBS alone. Injections were performed using a repeater pipette (Eppendorf, Hauppauge, NY) fitted with a $27 \mathrm{G} \times 1 / 2$ inch needle. Fish age at the time of challenge was 49 days post-hatch $(617$ temperature degree days).

Five fish were sampled per tank on day 1 and on day 5 postinjection for RNA extraction. Survival prior to and following sampling was monitored daily for 21 days with the exception of one PBS injected tank that was excluded on day 16 due to water failure. All fish were fed daily a standard commercial fishmealbased diet by hand (Ziegler Bros, Inc., USA). The day 1 sampled fish were removed prior to being fed and the day 5 sampled fish were removed after being fed.

\section{RNA EXTRACTION, LIBRARY PREPARATION, AND SEOUENCING}

The sampled fish were euthanized with $150 \mathrm{mg} \mathrm{mL}^{-1}$ tricaine methanesulfonate and individually flash frozen in liquid nitrogen and stored at $-80^{\circ} \mathrm{C}$. Total RNA was extracted from individual whole, ground fish using the standard TRIzol protocol (Invitrogen, Carlsbad, CA). Total RNA was extracted and integrity confirmed by running a $1 \%$ agarose gel. Equal amounts of RNA from five fish were pooled from each of the 12 tanks at each of the two time points (total of 24 pools, $n=120$ fish total). The cDNA libraries were prepared using Illumina's TruSeq Stranded mRNA Sample Prep kit following the manufacturer's instructions. Briefly, mRNA was selected with oligo(dT) beads and chemically fragmented to a size of $\sim 100-400 \mathrm{nt}$ before annealing of random hexamers and first strand cDNA synthesis. The 24 indexed and barcoded libraries were randomly divided into three groups (eight libraries per group) and sequenced in three lanes of an Illumina HiSeq 2000 (single-end, 100 bp read length) at the University of Illinois at Urbana-Champaign. All raw RNA-seq reads were submitted to the NCBI Short Read Archive under accession number BioProject ID PRJNA259860 (accession number SRP047070). RNA sequence reads that matched the F. psychrophilum CSF259-93 genome sequence (GenBank accession CP007627.1) were separated from host RNA-seq data and counted in each library.
Frozen fish homogenate lysates $(500 \mu \mathrm{L})$, stored at $-80^{\circ} \mathrm{C}$, were individually processed to isolate genomic DNA (TRIzol DNA Isolation Procedure) and qPCR detection of F. psychrophilum genomic DNA was performed as described previously (Marancik and Wiens, 2013). Bacterial genome equivalents were normalized to per $100 \mathrm{ng}^{-1}$ extracted DNA.

\section{GENES DESCRIBED AS REGULATED IN RESPONSE TO F. PSYCHROPHILUM CHALLENGE}

Based on a meta-analysis of studies in which rainbow trout were challenged with F. psychrophilum, 23 genes encoding immune relevant factors with putative roles in inflammation, innate disease response, and adaptive immunity were utilized as a curated gene reference set that included $c d 3, c d 8, m x-1$ (Overturf and LaPatra, 2006), saa (Villarroel et al., 2008), igm, igt, inf- $\gamma$, $i l-8$, tcr- $\beta$, tlr5, tnf- $\alpha$ (Evenhuis and Cleveland, 2012), $m t-a$, sod-1, tgf- $\beta$ (Orieux et al., 2013), and $c d 4, i l-1 \beta$, il-6, il-17c1, il-17c2, IL-4/13A, foxp3b, mhc-I, and $m h c-I I$ (Henriksen et al., 2014).

\section{RAINBOW TROUT REFERENCE GENOME, GO ANNOTATION, AND IDENTIFICATION OF IMMUNE RELEVANT GENES}

In addition to the curated reference gene dataset described above, the recently released rainbow trout genome sequence accession CCAF000000000 (Berthelot et al., 2014) encoding 46,585 predicted coding mRNA sequences were utilized as a more complete reference set of gene models. Briefly, the Onchorhynchus_mykiss_pep.fa file (April 24, 2014 release), accessed at (http://www.genoscope.cns.fr/trout-ggb/data), was subjected to Blast2GO sequence annotation pipeline with the default parameters applied in the blast, mapping, and annotation steps using a local Blast2GO database (GO database file go_201401-assocdb-data). NCBI non-redundant protein sequence (nr) database (build 12/13/2013) was used as the reference in the blast step. Of the 46,585 protein-coding gene models with supporting protein evidence from other vertebrates, a total of 46,103 were assigned a "Sequence Description" from the blast step and most of these predicted protein sequences assigned GO terms (Supplementary Data Sheet 1) from the Blast2GO annotation step. A subset of 2633 genes were identified as "putative immune relevant" either by sorting genes identified by GO annotation as "Immune Response" or by manual annotation based on sequence description (Supplementary Data Sheet 2). We excluded from analyses gene models encoding long non-coding RNAs and microRNAs. Splice variants were not analyzed and will be the subject of further study.

\section{IDENTIFICATION OF DIFFERENTIALLY EXPRESSED GENES}

To identify the differentially expressed genes, reads from each library were mapped against the annotated gene database and the coding sequences from the rainbow trout genome assembly. Based on the high quality score distribution of the RNA-seq reads, the whole $100 \mathrm{bp}$ of the sequences were used in this step. Bowtie short read aligner (Langmead et al., 2009) was used in mapping the reads to the references with a maximum of two mismatches allowed and no gaps. The output of Bowtie was filtered with an in-house Perl script to generate a count table, where a 
number at the corner of row $i$ and column $j$ represents the number of total mapped reads to the transcript $i$ from the library $j$. For principle component analyses (PCA), reads were normalized to reads per kilobase of exon model per million mapped reads (RPKM) using an in-house script (Gao, available upon request) using the formula described previously (Mortazavi et al., 2008).

$$
\begin{aligned}
R P K M & =N_{\text {read }} /\left(L_{\text {exon }} / 10^{3}\right) /\left(N_{\text {total }} / 10^{6}\right) \\
& =10^{9} N_{\text {read }} /\left(L_{\text {exon }} N_{\text {total }}\right)
\end{aligned}
$$

Where $N_{\text {read }}$ is defined as the number of reads mapped to the gene; $L_{\text {exon }}$ is defined as the total bases of the sequence of the gene, and $N_{\text {total }}$ is defined as the total number of reads mapped to the whole reference (sum of the $N_{\text {read }}$ for all genes). Raw data and normalized RNA-seq data $(R P K M)$ for all samples including immune relevant genes are included as Supplementary Data (Supplementary Data Sheet 3).

Principal component and nearest neighbor network analyses were performed using Qlucore Omics Explorer (v3.0). Read count data from the 24 samples (RPKM) were $\log _{2}$ transformed and subjected to normalization (mean $=0$ and variance $=1$ ) and variables (genes) subjected to multi-group comparison with a false discovery rate (FDR) $q<0.05\left(R^{2} \geq 0.36\right)$ and $p<0.002$ $\left[F_{(1,22)} \geq 12.42\right]$.

The raw count table was also input into the $\mathrm{R}$ package DESeq2 (Love et al., 2014), datasets selected for pair-wise comparisons, and the standard differential analysis steps of DESeq2 applied to the selected datasets. The output table of DESeq 2 contains a column of adjusted $p$-value (padj) obtained using the BenjaminiHochberg procedure (Benjamini and Hochberg, 1995), and we utilized a cut-off of $p_{a d j}<0.05$ as a criteria for differential expression with no filtering based on fold-change. For more stringent data filtering and visualization, data were first sorted by variance $2.5 \%$, then filtered by $q<0.01$ and $\log _{2}$ fold change of $>3$ (Qlucore Omics Explorer v3.0).

\section{GO ANNOTATION ENRICHMENT ANALYSES}

The GOSSIP program embedded in the Blast2GO package was used for GO enrichment analysis (Bluthgen et al., 2005a). This program examines each GO term for gene annotation enrichment by comparing a test set with a reference set using Fisher's exacttest method. In our analysis, the differentially expressed genes were selected as the test set and all the peptide sequences from the rainbow trout genome assembly for which GO terms were assigned, excluding the test set, were used as the reference set. FDR is computed by GOSSIP using an analytical equation (Bluthgen et al., 2005b) and the final list of the enriched GO terms selected at the FDR $<5 \%$.

\section{VALIDATION OF RNA-seq DATA BY qPCR}

Six genes and primer sets were chosen for qPCR analysis to determine fold-change differences between control and infected fish on day 5 post-infection (Table S1). Extracted RNAs were treated with Optimize ${ }^{\mathrm{TM}}$ DNAase I (Fisher Bio Reagents, Hudson, $\mathrm{NH})$ to remove contaminating genomic DNA. One microgram of DNAase treated RNA was used to make cDNA in a total reaction volume of $20 \mu \mathrm{L}$. cDNA was synthesized using the Verso cDNA Synthesis Kit (Thermo Scientific, Hudson, NH) following the manufacturer described protocol. Reverse transcription reaction was carried out using My Cycler ${ }^{\mathrm{TM}}$ Thermal Cycler (Bio Rad, Hercules, CA) at $42^{\circ} \mathrm{C}$ for $30 \mathrm{~min}$ (one cycle amplification) followed by $95^{\circ} \mathrm{C}$ for $2 \mathrm{~min}$ (inactivation). Anchored oligo(dT) primer, at a final concentration of $25 \mathrm{ng} / \mu \mathrm{L}$, was used to prime the reverse transcription reaction.

Relative gene expression was determined by CFX96 ${ }^{\mathrm{TM}}$ Real Time System (Bio Rad, Hercules, CA). Forward and reverse primer sequences were manually aligned to their respective genes for validation. cDNA amplification was performed using DyNAmo Flash SYBR Green Master Mix (Thermo Scientific, Hudson, $\mathrm{NH}$ ) containing $0.1 \mathrm{~nm} / \mu \mathrm{L}$ forward and reverse primers and $0.0025 \mu \mathrm{g} / \mu \mathrm{L}$ of cDNA template in a total reaction volume of $20 \mu \mathrm{L}$. Initial denaturation was done at $95^{\circ} \mathrm{C}$ for $7 \mathrm{~min}$. Fortythree cycles of amplification were carried out at the condition: $95^{\circ} \mathrm{C}$ for $0.1 \mathrm{~min}$ (denaturation), $57-64^{\circ} \mathrm{C}$ (annealing and extension) and $60^{\circ} \mathrm{C}$ for $5 \mathrm{~min}$ (final extension). Different annealing temperatures were used for different primers depending on their melting temperature (Table S1).

Real time data were analyzed using the software Bio-Rad CFX Manager (Bio Rad, Hercules, CA). Differential gene expression was calculated using the standard curve method in which $\beta$-actin (Accession: AJ438158) was used as endogenous reference to normalize the target gene. $\beta$-actin expression levels demonstrated in RNA-seq data were similar in PBS and F. psychrophilum-injected fish (data not shown). qPCR data were quantified using delta delta Ct $(\Delta \Delta \mathrm{Ct})$ methods (Schmittgen and Livak, 2008). Ctvalues of $\beta$-actin were subtracted from Ct-values of the target gene to calculate the normalized value $(\Delta \mathrm{Ct})$ of the target gene in both the calibrator samples (PBS-injected) and test samples (F. psychrophilum-injected). The $\Delta \mathrm{Ct}$ value of the calibrator sample was subtracted from the $\Delta \mathrm{Ct}$ value of the test sample to get the $\Delta \Delta \mathrm{Ct}$ value. Fold change in gene expression in the test sample relative to the calibrator sample was calculated by the formula $2^{-\Delta \Delta C t}$ and the normalized target $\mathrm{Ct}$ values in each infected and non-infected group was averaged. Correlation between gene expression fold-change measured by qPCR and RNA-seq was performed by Pearson correlation. All statistics were performed with a significance of $P<0.05$.

\section{RESULTS}

DISEASE RESISTANCE PHENOTYPE AND F. PSYCHROPHILUM LOAD IN THREE GENETIC LINES FOLLOWING EXPERIMENTAL CHALLENGE

Following injection challenge, survival significantly differed between the three genetic lines $(P<0.001)$ with the ARS-Fp-R line exhibiting highest survival (92.3\%), the ARS-Fp-C line intermediate survival (54.6\%), and the ARS-Fp-S line exhibiting the lowest survival (29.4\%) (Figure 1A). The 62.9 percentage point survival difference between the ARS-Fp-R and ARS-Fp-S lines is consistent with the expected percentage point survival difference calculated from estimated parental breeding values (data not shown), analyzed as previously described (Leeds et al., 2010). Survival of PBS injected fish was $>98 \%$ per line over the 21 days challenge period. Whole body Fp loads, measured by qPCR, 


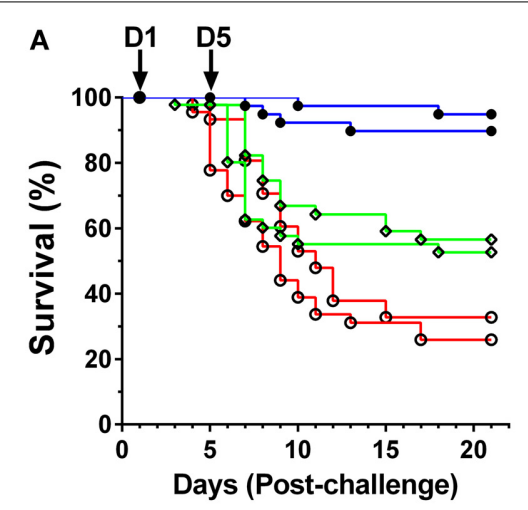

B

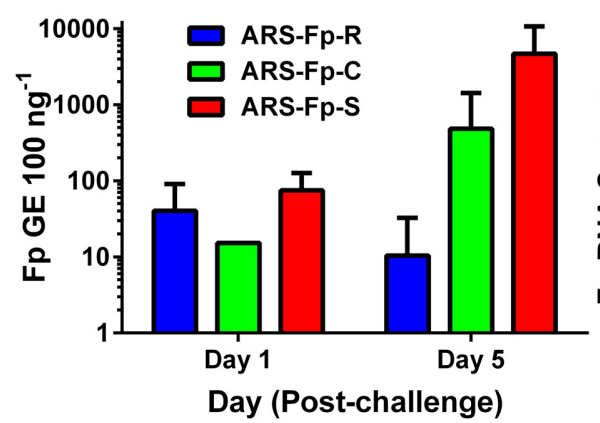

C

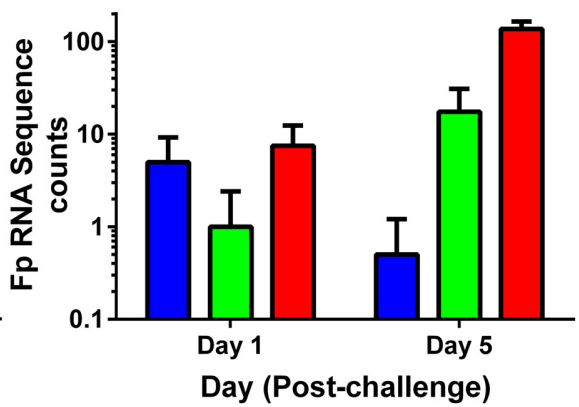

FIGURE 1 | (A) Post-challenge survival of ARS-Fp-R line (resistant, blue line), ARS-Fp-C line (control, green line), and ARS-Fp-S line (susceptible, red line) fish, challenged using replicate tanks. Survival differences were significant between genetic lines $(P<0.001)$. Fish were injected with either F. psychrophilum in PBS ( $n=100$ fish per line) or with PBS alone (data not shown) and survival monitored for 21 days. Five fish were sampled from each tank ( $n=10$ total) on days 1 and 5 post-challenge (arrows) for RNA-seq analysis. (B) Mean F. psychrophilum load, genome equivalents per $100 \mathrm{ng}$ extracted DNA (+1 SD) measured by qPCR. Individual fish were tested ( $n=10$ fish per group) with the exception of day 1 ARS-Fp-C line $(n=1)$ as samples were not available. Load differences were significantly different between genetic lines on day 5 $(P<0.001)$. (C) Mean $F$. psychrophilum cDNA count per library $(+1 \mathrm{SD})$ identified from the RNA-seq dataset. demonstrated no difference between genetic lines on day 1 but significant differences on day $5(P<0.001)$, with ARS-Fp-R line mean loads of $10 \pm 22 \mathrm{~F}$. psychrophilum genome equivalents (GE) ( $n=10$ fish, $\pm 1 \mathrm{SD}$ ), increasing to $484 \pm 937 \mathrm{GE}$ in the ARS-Fp$\mathrm{C}$ line $(n=10, \pm 1 \mathrm{SD})$, and highest mean load was present in the ARS-Fp-S line $4683 \pm 6011 \mathrm{GE}(n=10, \pm 1 \mathrm{SD})$ (Figure 1B). In the RNA-seq libraries, a small number of sequences were present that matched the F. psychrophilum CSF259-93 genome. These presumably represent either cDNA from bacterial expressed genes or contaminating genomic DNA. The abundance of the F. psychrophilum read counts were similar between genetic lines on day 1 , and by day 5, lowest in ARS-Fp-R line fish and highest in ARSFp-S line fish (Figure 1C). In summary, by day 5 post-challenge the phenotypes of the ARS-Fp-R, ARS-Fp-C and ARS-Fp-S lines displayed expected differences in pathogen load and subsequently post-challenge mortality.

\section{RAINBOW TROUT RNA-seq REFERENCE DATASET, GO ANNOTATION, AND MANUAL IMMUNE GENE CURATION}

A total of 520 million sequence reads were generated from the 24 libraries with an average of 21.7 million (M) RNA-seq reads per library ranging from $17.4 \mathrm{M}$ to $24.4 \mathrm{M}$ reads (Table 1 ). Approximately half of the reads aligned to the reference transcriptome coding sequence, averaging $11.2 \mathrm{M}(51.8 \%)$ per library with a range of $9.3 \mathrm{M}-12.5 \mathrm{M}$. Of the 46,585 genes identified in the rainbow trout genome having protein coding evidence, an average of 43,068 (92.4\%), exhibited detectable levels of expression, defined as $>1$ sequence read per gene (Table 1 ). The number of genes with an average of $\geq 10$ read counts across the 24 libraries fell to an average of $32,830(70.5 \%)$. In order to normalize gene expression across samples, data were converted to reads per kilobase of exon model per million mapped reads (RPKM) format (Supplementary Data Sheet 3). Of the putative immune relevant genes, $1797(68.2 \%)$ had an average of $\geq 1$ RPKM across the 24 libraries (Supplementary Data Sheet 3).

\section{GLOBAL TRANSCRIPT ABUNDANCE DIFFERENCES BETWEEN CONTROL AND INFECTED AND BY SAMPLE DAY}

Across the complete dataset, comparison of F. psychrophilum infected vs. PBS injected groups by principal component and nearest neighbor network analysis identified samples grouped by day and infection status (Figure 2). Most tank replicates were connected by nearest-neighbor analysis although there was variation observed between tanks. The replicate tanks for the day 5 PBS injected ARS-Fp-S line (red colored balls), day 1 F. psychrophilum infected ARS-Fp-R line (blue colored balls), and day 5 F. psychrophilum ARS-Fp-C line (green colored balls) grouped together but were not directly connected by the network analysis.

\section{DIFFERENTIALLY EXPRESSED GENES COMMON TO THE THREE GENETIC LINES IN RESPONSE TO F. PSYCHROPHILUM INFECTION}

Two-group comparison by infection (Qlucore) identified a total of 1884 genes as differentially regulated $(q<0.05)$ accounting for approximately $4.04 \%$ of the coding genes identified in the genome (see Table S2, Qlucore $\boldsymbol{q}<\mathbf{0 . 0 5}$ for the complete list). Of the differentially regulated genes, 279 (14.8\%) were categorized as immune relevant by GO or manual annotation. In order to more precisely identify differences between samples, 24 pairwise comparisons were tabulated by DESeq2 using a $q<0.05$ (Table 2). There was a decrease in the number of differentially regulated genes in the infected ARS-Fp-R line fish compared to PBS injected fish between day 1 ( $n=515$ genes) and day 5 ( $n=$ 428 genes) time points. In contrast, the number of differentially regulated genes in the infected ARS-Fp-C line fish compared to PBS injected fish, increased from day 1 ( $n=20$ genes) to day 5 ( $n=2201$ genes). The number of differentially regulated genes in the infected ARS-Fp-S line fish compared to PBS injected fish increased from day $1(n=1663)$ to day $5(n=2225)$. In general, there were relatively few differentially regulated genes between the three PBS injected genetic lines, ranging from 3 to 246 genes (Table 2). Bacterial challenge increased the number 
Table 1 | Summary statistics of 24 RNA-seq libraries listed by genetic line, time, infection status, and tank replicate.

\begin{tabular}{|c|c|c|c|c|c|c|c|c|}
\hline Genetic line & Day & $\begin{array}{l}\text { Infection, } \\
\text { Tank }\end{array}$ & $\begin{array}{c}\text { Biosample } \\
\text { Accession No }\end{array}$ & Total reads & $\begin{array}{l}\text { Mapped } \\
\text { reads }\end{array}$ & $\begin{array}{l}\text { Percentage } \\
\text { reads mapped }\end{array}$ & $\begin{array}{c}\text { Uniquely } \\
\text { mapped reads }\end{array}$ & $\begin{array}{c}\text { Number of } \\
\text { expressed genes }\end{array}$ \\
\hline \multirow[t]{6}{*}{ ARS-Fp-R } & 1 & $\mathrm{Fp}, \mathrm{Tk} 25$ & SAMN03014722 & $20,061,852$ & $10,210,344$ & $50.89 \%$ & $9,552,696$ & 42,919 \\
\hline & \multirow[t]{2}{*}{5} & $\mathrm{Fp}, \mathrm{Tk} 25$ & SAMN03014726 & $21,409,329$ & $10,973,051$ & $51.25 \%$ & $10,445,614$ & 43,000 \\
\hline & & $\mathrm{Fp}, \mathrm{Tk} 26$ & SAMN03014727 & $23,958,681$ & $11,795,961$ & $49.23 \%$ & $11,135,354$ & 43,654 \\
\hline & 1 & PBS, Tk27 & SAMN03014724 & $22,129,914$ & $11,745,879$ & $53.08 \%$ & $10,998,947$ & 43,303 \\
\hline & \multirow[t]{2}{*}{5} & PBS, Tk27 & SAMN03014728 & $24,361,298$ & $12,124,063$ & $49.77 \%$ & $11,507,535$ & 43,446 \\
\hline & & PBS, Tk28 & SAMN03014729 & $23,318,224$ & $11,741,589$ & $50.35 \%$ & $11,171,392$ & 43,504 \\
\hline \multirow[t]{4}{*}{ ARS-Fp-C } & \multirow[t]{2}{*}{1} & $\mathrm{Fp}, \mathrm{Tk} 33$ & SAMN03014738 & $20,940,097$ & $11,050,478$ & $52.77 \%$ & $10,225,748$ & 43,229 \\
\hline & & $\mathrm{Fp}, \mathrm{Tk} 34$ & SAMN03014739 & $19,151,755$ & $10,393,600$ & $54.27 \%$ & $9,629,194$ & 42,162 \\
\hline & 5 & Fp, Tk33 & SAMN03014742 & $21,117,398$ & $10,817,377$ & $51.22 \%$ & $10,299,653$ & 42,904 \\
\hline & 5 & PBS, Tk36 & SAMN03014745 & $20,535,582$ & $10,422,413$ & $50.75 \%$ & $9,824,416$ & 43,013 \\
\hline \multirow[t]{8}{*}{ ARS-Fp-S } & \multirow[t]{2}{*}{1} & Fp, Tk29 & SAMN03014730 & $17,389,164$ & $9,377,592$ & $53.93 \%$ & $8,617,417$ & 42,438 \\
\hline & & $\mathrm{Fp}, \mathrm{Tk} 30$ & SAMN03014731 & $21,095,859$ & $11,103,652$ & $52.63 \%$ & $10,198,695$ & 42,765 \\
\hline & \multirow[t]{2}{*}{5} & Fp, Tk29 & SAMN03014734 & $21,467,982$ & $11,073,977$ & $51.58 \%$ & $10,409,769$ & 43,263 \\
\hline & & $\mathrm{Fp}, \mathrm{Tk} 30$ & SAMN03014735 & $23,427,268$ & $11,922,324$ & $50.89 \%$ & $11,172,000$ & 43,479 \\
\hline & \multirow[t]{2}{*}{1} & PBS, Tk31 & SAMN03014732 & $20,622,290$ & $11,310,300$ & $54.85 \%$ & $10,482,544$ & 42,903 \\
\hline & & PBS, Tk32 & SAMN03014733 & $24,139,642$ & $12,522,402$ & $51.87 \%$ & $11,594,067$ & 43,561 \\
\hline & \multirow[t]{2}{*}{5} & PBS, Tk31 & SAMN03014736 & $22,371,928$ & $11,319,845$ & $50.60 \%$ & $10,753,477$ & 43,607 \\
\hline & & PBS, Tk32 & SAMN03014737 & $22,338,190$ & $11,425,396$ & $51.15 \%$ & $10,750,682$ & 43,319 \\
\hline
\end{tabular}

Total number of $100 \mathrm{bp}$ reads and the number that match reference genes are listed.

${ }^{a}$ The number of expressed genes is defined as genes having at least one read count with 2 bp or less mismatch and no gaps ( $n=46,585$ reference genes).

of differentially expressed genes between genetic lines from day 1 to day 5 in all between-line comparisons. There was a strong sampling time effect within each line including PBS injected groups possibly due to differences in feeding or post-injection recovery.

Analysis of GO term enrichment within the pair-wise comparisons revealed a larger number of over-represented terms within the dataset as compared to under-represented terms (Table 3 ). Overrepresented processes in the ARS-Fp-S line included defense response to bacterium, inflammatory response, leukotriene and arachidonic acid production, complement activation, humoral immune response, antigen processing, chemotaxis, B cell homeostasis, interleukin-2 mediated pathway signaling and cellular iron homeostasis (Supplementary Data Sheet 5 for complete list). The ARS-Fp-R line Day 1 GO term enrichment included several categories that include response to wounding and wound healing and was enriched for cytokine and chemokine activity. ARS-Fp-R line day $5 \mathrm{GO}$ term enrichment included complement activation, inflammatory response, B cell mediated immunity and defense response to bacterium.

Pair-wise analysis of differentially regulated genes shared between infected and PBS injected fish within each sampling day identified no common genes on day 1, although 274 were shared between ARS-Fp-R and ARS-Fp-S line fish (Figure 3). This may in part be due to the low numbers of genes differentially expressed between the ARS-Fp-C line replicates. Among all day five samples, 175 common genes were differentially regulated, by pair-wise comparisons, in all three lines (see Table S2, Pair-wise for the complete list). Of these, the majority $(89 \%, n=156)$ were upregulated, while only 19 were consistently downregulated. Application of stringent data filtering to the entire dataset (Qlucore, $q=0.01$ and $>3$-fold $\log _{2}$ expression difference) identified 110 genes that were the most robust predictors of infection status that collapsed to 51 common sequence descriptions (Figure 4 and Table S2: tab Qlucore $q<0.01, \log _{2}>3$ fold for complete gene list). Many shared coordinated patterns of gene expression across samples were identified by unsupervised hierarchal clustering (Figure 4). The most highly regulated immune relevant genes included serum amyloid A (GSONMT 00016296001, GSONMT00005013001), complement clq-like protein 2-like (GSONMT00002696001), differentially regulated trout protein 1 precursor (GSONMT00048193001, GSONMT 00025517001), leukocyte cell-derived chemotaxin 2 precursor (GSONMT00024746001, GSONMT00065856001), toll-like receptor 5 membrane form (GSONMT00013855001), c-type lectin domain family 4 member e (GSONMT00005166001), c 


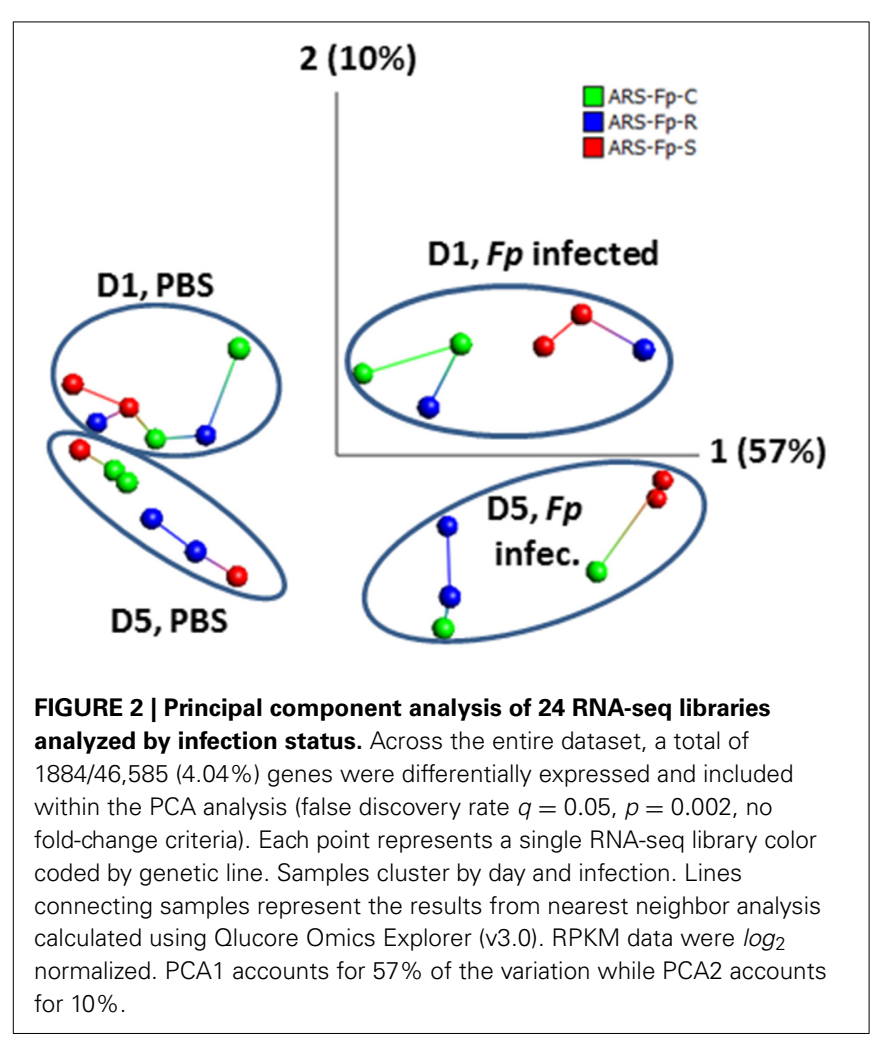

type lectin receptor b (GSONMT00023806001), cd59b glycoprotein (GSONMT00025518001), and interleukine-1 receptor type 2-like (GSONMT00066304001). Interestingly, a number of putative metabolic genes were also highly expressed in infected ARS-Fp-S line fish compared to the ARS-Fp-R line including cis-aconitate decarboxylase-like (GSONMT00057407001), tbtbinding partial (GSONMT00003889001), l-serine dehydratase l-threonine deaminase-like (GSONMT00025010001), leptin (GSONMT00002603001), and growth differentiation factor 15 (GSONMT00000024001), catechol o-methyltransferase domaincontaining protein 1-like (3 genes) and hepcidin (GSONMT 00082379001). Multiple paralogues of several less abundantly expressed immune relevant genes included complement component 3 (4 genes), interferon-induced guanylate-binding protein 1 (3 genes), interferon-induced guanylate-binding protein 1-like (5 genes), interferon-induced protein 44-like (3 genes) and microfibril-associated glycoprotein 4-like (3 genes). Differentially regulated cytokine genes included interleukin 11 (GSONMT00009406001) and interleukin 1-beta (GSONMT00005489001) as well as nine chemokine genes that included: cc chemokine (GSONMT00017873001, GSONMT 00007278001), c-c motif chemokine 19 precursor (GSONMT 00014841001, GSONMT00057082001), c-c motif chemokine 4-like (GSONMT00080018001), chemokine ck-1 (GSONMT 00024124001), cxc chemokine (GSONMT00080684001), and interleukin 8 (GSONMT00038968001, GSONMT00059090001). Immune genes of interest also included, programmed cell death 1 ligand-1 (GSONMT00040812001), tnf receptor superfamily member 5 (GSONMT00012579001), tnf receptor superfamily member 6b (GSONMT00034343001) and tnf receptor
Table 2 | Summary of pairwise comparison between treatment groups including infection status, genetic line, and day $(q \leq 0.05)$.

\begin{tabular}{|c|c|c|}
\hline Comparisons & $\begin{array}{l}\text { Day, Genetic line, } \\
\text { and Infection status }{ }^{a}\end{array}$ & $\begin{array}{c}\text { Diff. } \\
\text { expressed } \\
\text { genes }\end{array}$ \\
\hline \multirow[t]{6}{*}{ Infected vs. PBS } & Day1 R-line (Fp) vs. R-line (PBS) & 515 \\
\hline & Day5 R-line (Fp) vs. R-line (PBS) & 428 \\
\hline & Day1 C-line (Fp) vs. C-line (PBS) & 20 \\
\hline & Day5 C-line (Fp) vs. C-line (PBS) & 2201 \\
\hline & Day1 S-line (Fp) vs. S-line (PBS) & 1663 \\
\hline & Day5 S-line (Fp) vs. S-line (PBS) & 2225 \\
\hline \multirow[t]{6}{*}{ Genetic lines-PBS } & Day1 R-line (PBS) vs. S-line (PBS) & 76 \\
\hline & Day1 R-line (PBS) vs. C-line (PBS) & 3 \\
\hline & Day1 S-line (PBS) vs. C-line (PBS) & 28 \\
\hline & Day5 R-line (PBS) vs. S-line (PBS) & 45 \\
\hline & Day5 R-line (PBS) vs. C-line (PBS) & 246 \\
\hline & Day5 S-line (PBS) vs. C-line (PBS) & 61 \\
\hline \multirow[t]{6}{*}{ Genetic lines- $F p$ Inf. } & Day1 R-line (Fp) vs. S-line (Fp) & 150 \\
\hline & Day5 R-line (Fp) vs. S-line (Fp) & 1016 \\
\hline & Day1 R-line (Fp) vs. C-line (Fp) & 28 \\
\hline & Day5 R-line (Fp) vs. C-line (Fp) & 159 \\
\hline & Day1 S-line (Fp) vs. C-line (Fp) & 37 \\
\hline & Day5 S-line (Fp) vs. C-line (Fp) & 1758 \\
\hline \multirow[t]{6}{*}{ Between time points } & Day5 vs. Day1 R-line (PBS) & 1286 \\
\hline & Day5 vs. Day1 C-line (PBS) & 294 \\
\hline & Day5 vs. Day1 S-line (PBS) & 376 \\
\hline & Day5 vs. Day1 R-line (Fp) & 334 \\
\hline & Day5 vs. Day1 C-line (Fp) & 2469 \\
\hline & Day5 vs. Day1 S-line (Fp) & 2434 \\
\hline
\end{tabular}

a Abbreviations: R, ARS-Fp-R; C, ARS-Fp-C; S, ARS-Fp-S; Fp, F. psychrophilum challenged; $P B S$, phosphate buffered saline injected.

superfamily member 9 (GSONMT00057532001), and b-cell receptor cd22-like isoform $\mathrm{x} 2$ (GSONMT00072668001).

\section{GENE EXPRESSION IN NAIVE FISH}

Gene expression profiles were compared in PBS-injected ARSFp-R, ARS-Fp-C and ARS-FP-S line fish to examine the effect of selective breeding on the transcriptome of naive animals. In the first analysis, pair-wise comparisons between lines were examined. On day 1 post challenge, three genes demonstrated significantly different transcript abundance between PBS-injected ARS-Fp-R and ARS-Fp-C fish, 76 genes were different between the ARS-Fp-R and ARS-Fp-S line fish, and 28 genes were different between the ARS-Fp-C and ARS-Fp-S line fish (Table 2 and Supplementary Data Sheet 4: tabs R_C PBS Day 1, R_S PBS Day 1, and S_C PBS Day 1, respectively). None of these genes were common to all three comparisons and none were found to be similarly differentially regulated between genetic lines after challenge with F. psychrophilum. On day 5 post injection, the number of differentially regulated genes in naive fish increased with 246 genes demonstrating significant differences in transcript counts between the ARS-Fp-R and ARS-Fp-C lines, 45 genes between the 
Table 3 | GO enrichment categories determined from pair-wise comparison.

\begin{tabular}{lccc}
\hline Pair-wise comparison $^{a}$ & Total & Over-represented & Under-represented \\
\hline GORich_Fp_PBS_D1_R & 53 & 50 & 3 \\
GORich_Fp_PBS_D1_C & 11 & 11 & 0 \\
GORich_Fp_PBS_D1_S & 361 & 218 & 143 \\
GORich_Fp_PBS_D5_R & 326 & 156 & 170 \\
GORich_Fp_PBS_D5_C & 909 & 611 & 298 \\
GORich_Fp_PBS_D5_S & 607 & 333 & 274
\end{tabular}

${ }^{a}$ Abbreviations: $F p, F$. psychrophilum challenged vs. PBS, phosphate buffered saline injected; D1, day1; D5, day5; $R, A R S-F p-R$ line; C, ARS-Fp-C line; S, ARSFp-S line.

ARS-Fp-R and ARS-Fp-S lines, and 61 genes between the ARSFp-C and ARS-Fp-S lines (Table 2). No genes were common to all three comparisons and eight genes exhibited a significant difference within two comparisons with similar trends in infected fish (Supplementary Data Sheet 4). Of these genes, only complement factor h-like (GSONMT00015052001) has a purported immunologic role. Fold gene expression differences were 2.8 and 2.1 between naive resistant and susceptible and infected resistant and susceptible fish, respectively. Complement factor h-like was 1.8 fold-higher in naive control fish compared to naive susceptible fish and 5.0 fold different between the infected lines (Supplementary Data Sheet 4).

A global analysis of expression differences between lines was performed on the entire dataset. In these analyses, data were first filtered for variance $\geq 2.5 \%$ and then subjected to $q<0.05$ and infection status was removed as a factor. Within the complete dataset, 21 differentially expressed genes were identified (Figure 5). Several genes exhibited the pattern of low expression in ARS-Fp-S line, intermediate expression in ARS-Fp-C line and highest expression in ARS-Fp-R line. These included immune relevant genes tnf receptor superfamily member 14b-like isoform $\mathrm{x} 1$ and interleukine-1 receptor-like 1, genes. We also analyzed only day 1 samples, and identified additional immune relevant candidates that included complement clq-like protein 4 precursor, protein nlrc3-like and gata-binding factor 2-like isoform $\mathrm{x} 2$ (Figure 6). Interestingly, there were also genes that exhibited the opposite expression pattern: higher in ARS-Fp-S line and lower in the ARS-Fp-C and ARS-Fp-R lines. This included putative immune relevant gene nlrc5-partial (Figure 5) and ig-like v-type domain-containing protein family 187a-like (Figure 6).

\section{MANUAL EXAMINATION OF KNOWN IMMUNE GENES EXPRESSED IN RESPONSE TO F. PSYCHROPHILUM INFECTION}

In order to compare this dataset to previous analyses of gene expression following bacterial challenge, 23 known immune relevant genes were selected and de novo sequence alignment of the complete RNA-seq dataset was performed. There was a significant difference in transcript abundance between $F$. psychrophilum and PBS-challenged fish on day 1 and/or day 5 post-infection for 10/23 published gene sequence that were searched against the RNA-seq data set (Table 4) $\left(P_{\text {adj }}<0.05\right)$. Seven genes demonstrated a significant difference in transcript counts between

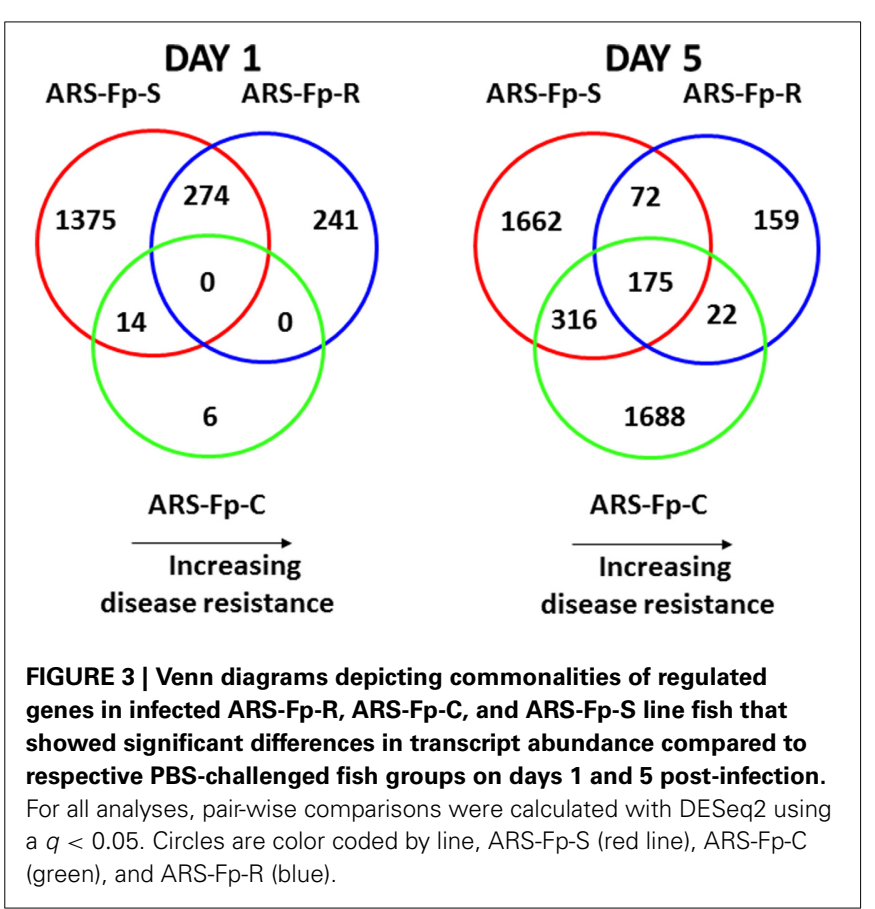

genetic lines on day 5 post-infection, with no significant differences occurring between genetic lines on day 1 (Table 4$)\left(P_{a d j}<\right.$ $0.05)$. There was no significant difference in transcript abundance between infected and control fish or between genetic lines for $\operatorname{tgf}-\beta, \operatorname{tcr}-\beta$, sodl $, m x-1, m t-a, m h c-1, i l-6, i l-4 / 13 A$, and foxp $3 b$. Transcript counts were too low to in at least one comparison to provide a significant $P_{a d j}$ value for $t n f-\alpha$, ill- $\beta, i l-17 c 2$, and inf- $\gamma$.

\section{VALIDATION OF RNA-seq DATA BY qPCR}

In order to begin validating RNA-seq transcript abundance estimates, selected differentially expressed genes were identified for qPCR analysis (Table S1). There was significant correlation between transcript fold-change values determined by RNA-seq and $\mathrm{qPCR}$ in control and infected fish on day 5 post-infection $(R=0.75, P<0.001)($ Figure 7$)$.

\section{DISCUSSION}

To our knowledge this is the first study describing wholebody transcriptome analysis and comparison of three divergently selected lines of rainbow trout exhibiting graded survival differences in response to standardized F. psychrophilum challenge. We identified large numbers of differentially expressed genes between genetic lines that increased in number with time and bacterial load. Since we utilized whole fish in these experiments, we interpreted differences in transcript abundance as upregulation/downregulation of genes or alteration of transcript stability. Importantly, the use of entire fish for RNA extraction rules out the potential for cell migration to confound expression differences inherent in studies utilizing defined immune tissue source (i.e., spleen, blood, or anterior kidney). A limitation of our approach is the likelihood of missing differentially expressed genes expressed only in rare subsets of cells. Nevertheless, our 


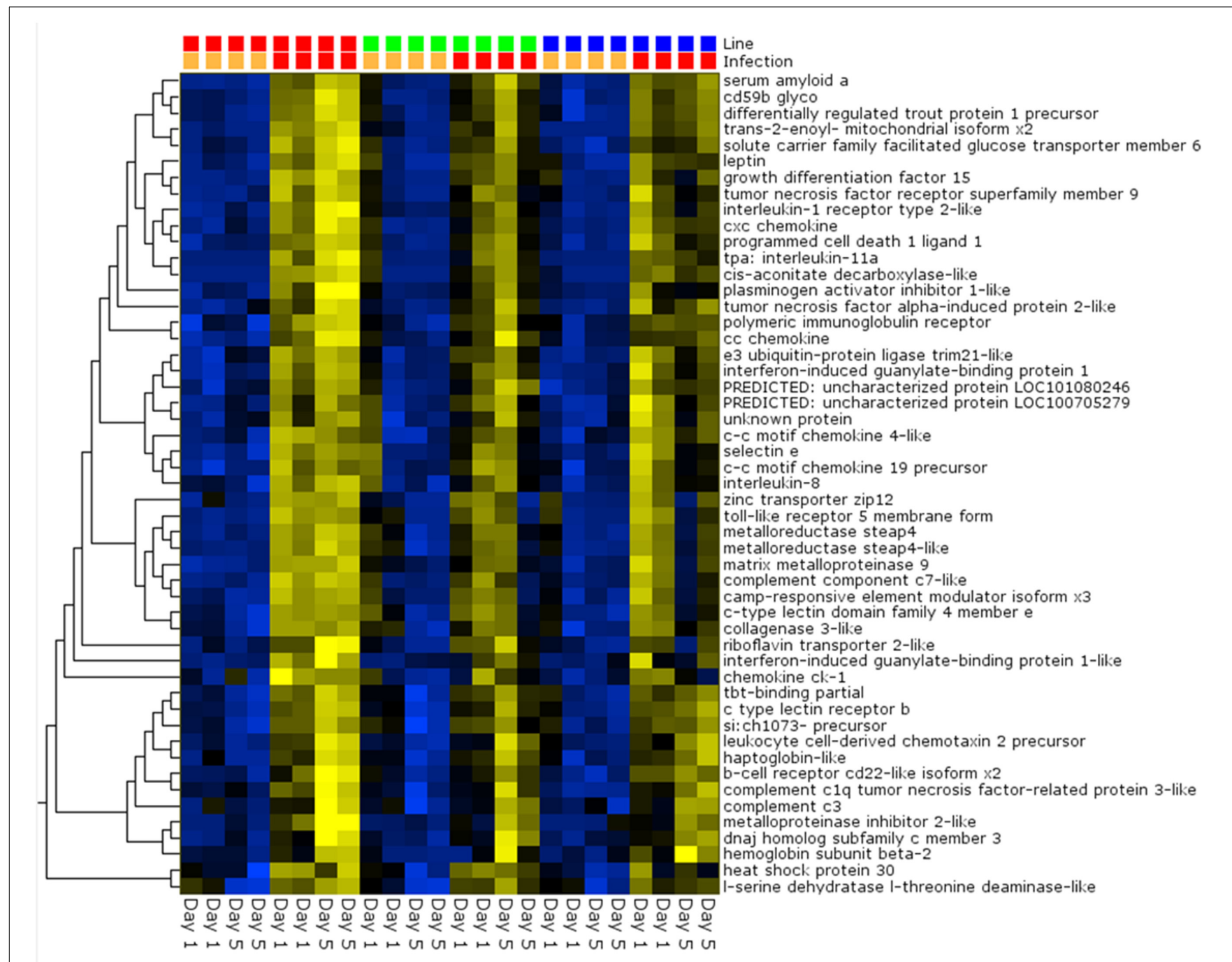

FIGURE 4 | Heat map of PCA analysis showing the most highly regulated genes in infected vs. day-matched PBS injected fish ( $q=0.01$ and $>\log _{2} 3$-fold cut-off). Genetic line (S-line red color, C-line green color,
R-line blue color) and infection status (PBS injected orange color, Fp injected red color) are shown on top and day post-infection is shown on bottom. Variables (genes) are grouped by hierarchal clustering. depth of sequence coverage allowed quantification of transcript abundance from about $70.5 \%$ of all identified protein coding genes identified within the rainbow trout genome and approximately $68.2 \%$ of putative immune relevant genes that we identified by automated and manual annotation. Genes identified as differentially expressed were primarily associated with the acute phase response to bacterial infection but we also identified genes associated with innate and adaptive immune responses, physiologic and metabolic processes, and wound healing. This experimental design allowed for inclusion of both mucosal and systemic immune system tissue sampling and demonstrates previously unrecognized changes in gene transcription that occur with BCWD. These findings illustrate the high degree of transcriptional complexity involved in the rainbow trout BCWD response and provide a reference data set to begin to understand the impact of selective breeding on the genetic basis of disease resistance.

\section{INDUCTION OF THE ACUTE PHASE RESPONSE AND INNATE IMMUNITY} The transcriptional response included induction of complement factors, acute-phase proteins, cytokines, chemokines and other genes associated with innate immunity. A relatively high number of complement factors were identified as upregulated after infection. This included multiple transcript alignments to genes encoding complement c3, complement c9, complement c4-b-like, and complement clq-like proteins. Generation of complement during the acute-phase response has been well-described in rainbow trout and likely imparts direct bactericidal activity (Sunyer and Lambris, 1998; Whyte, 2007). The magnitude and range of complement factor expression suggests the complement system constitutes an important aspect of the host response to F. psychrophilum although factors were not found to be significantly different between resistant and susceptible fish. A wide range of acute phase protein encoding genes, including serum 


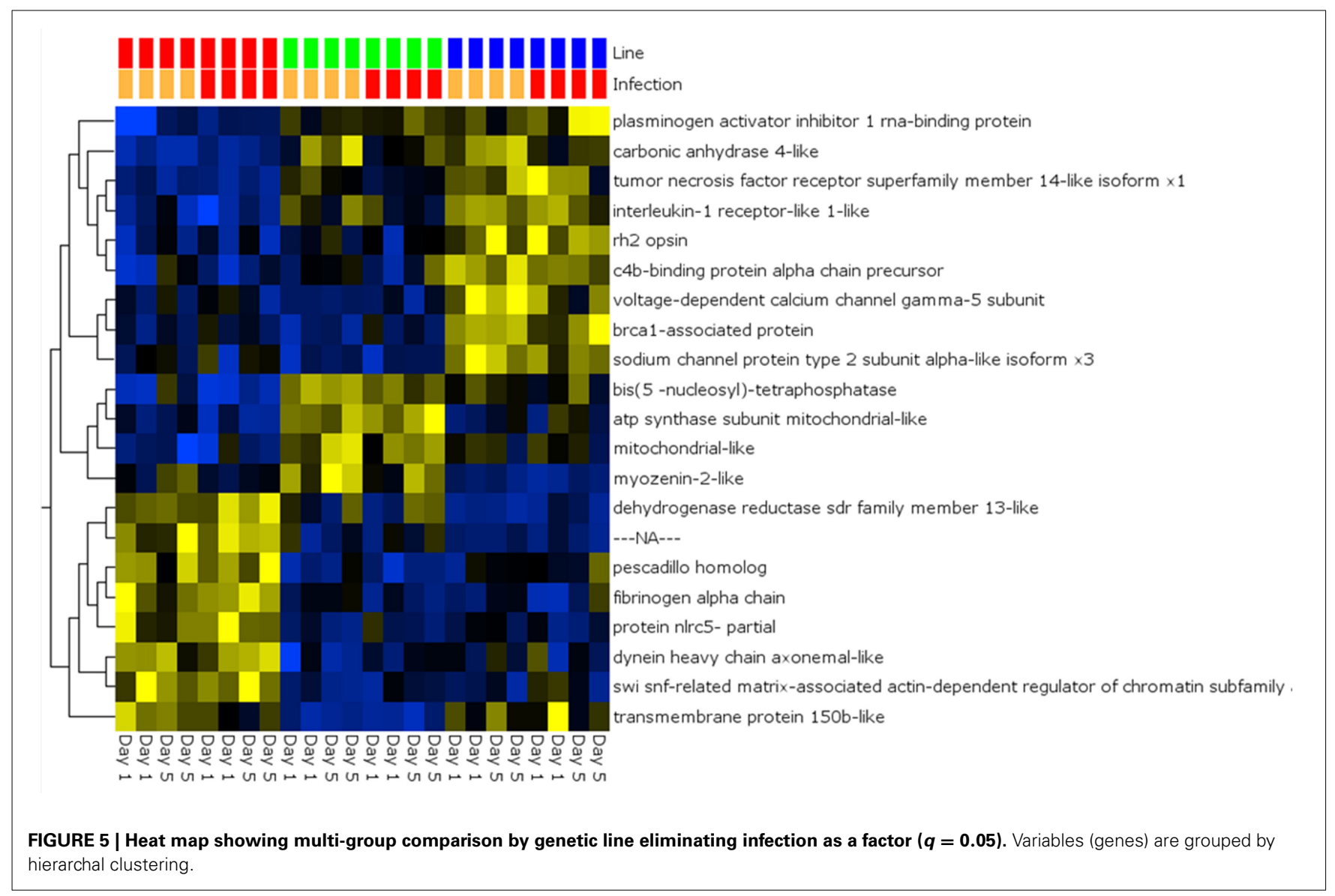

amyloid A and tlr5 were identified as highly expressed after infection. Similar trends have been described from tissues of rainbow trout affected by BCWD (Overturf and LaPatra, 2006; Langevin et al., 2012). Differentially expressed trout protein is a relatively recently recognized immune factor shown to be expressed during the salmonid acute phase response after bacterial infection (Bayne et al., 2001; Tsoi et al., 2004). Along with IL-1, IL-11, and IL-17-c1, these factors likely exert diverse pro-inflammatory and defensive actions including recruitment of inflammatory cells and further amplification of the acute phase response (Stadnyk, 1994; Jorgensen et al., 2001; Carrington et al., 2004; Wang et al., 2009). Significantly higher transcript counts for acute phase proteins and cytokines in the ARS-Fp-S line on day 5 may represent higher induction of pro-inflammatory conditions compared to the ARS-Fp-R line that correlates with higher bacterial loads.

\section{DIFFERENTIAL EXPRESSION OF GENES THAT CONTRIBUTE TO ADAPTIVE IMMUNITY}

A limited number of differentially regulated genes were identified that may be associated with adaptive immune processes. There was modest up-regulation of igm and igt genes and cellular factors associated with cell signaling and activation including $m h c-I I, c d 3, c d 4$, and $c d 8$ genes. Higher igm gene transcript levels in resistant and control-line fish compared to the susceptible-line suggest an earlier development of antibody mediated processes, although the converse pattern was observed for $m h c-I I$ gene transcript counts on the same days postinfection.

\section{GENES INVOLVED IN WOUND HEALING}

A number of genes associated with wound healing and wound response showed significant expression differences between naive and infected fish but no trends between genetic lines or sample day. These included syndecan-4-like isoform x2, plasminogen activator inhibitor 1-like, and ras-related c3 botulinum toxin substrate 2 precursor. All have roles in localized tissue repair in mammals through augmenting extracellular matrix reorganization, cellular growth and proliferation, and regulation of cell signaling (Romer et al., 1996; Woods and Couchman, 1998; Lin et al., 2005; Ojha et al., 2008). A limited number of genes with described roles in wound healing in other species (i.e., abhydrolase domain-containing protein 2-a-like and a collagen alpha- 1 chain-like factor) demonstrated reduced expression in infected fish. Wound repair has not been wellcharacterized in fish and the general effect of these genes on the host response to infection is unknown. Necrosis of internal organs, peripheral skeletal muscle and skin is likely directly associated with host morbidity and mortality (Nilsen et al., 2011; Marancik et al., 2014b) and further studies are needed to determine how wound healing impacts recovery and survival. 


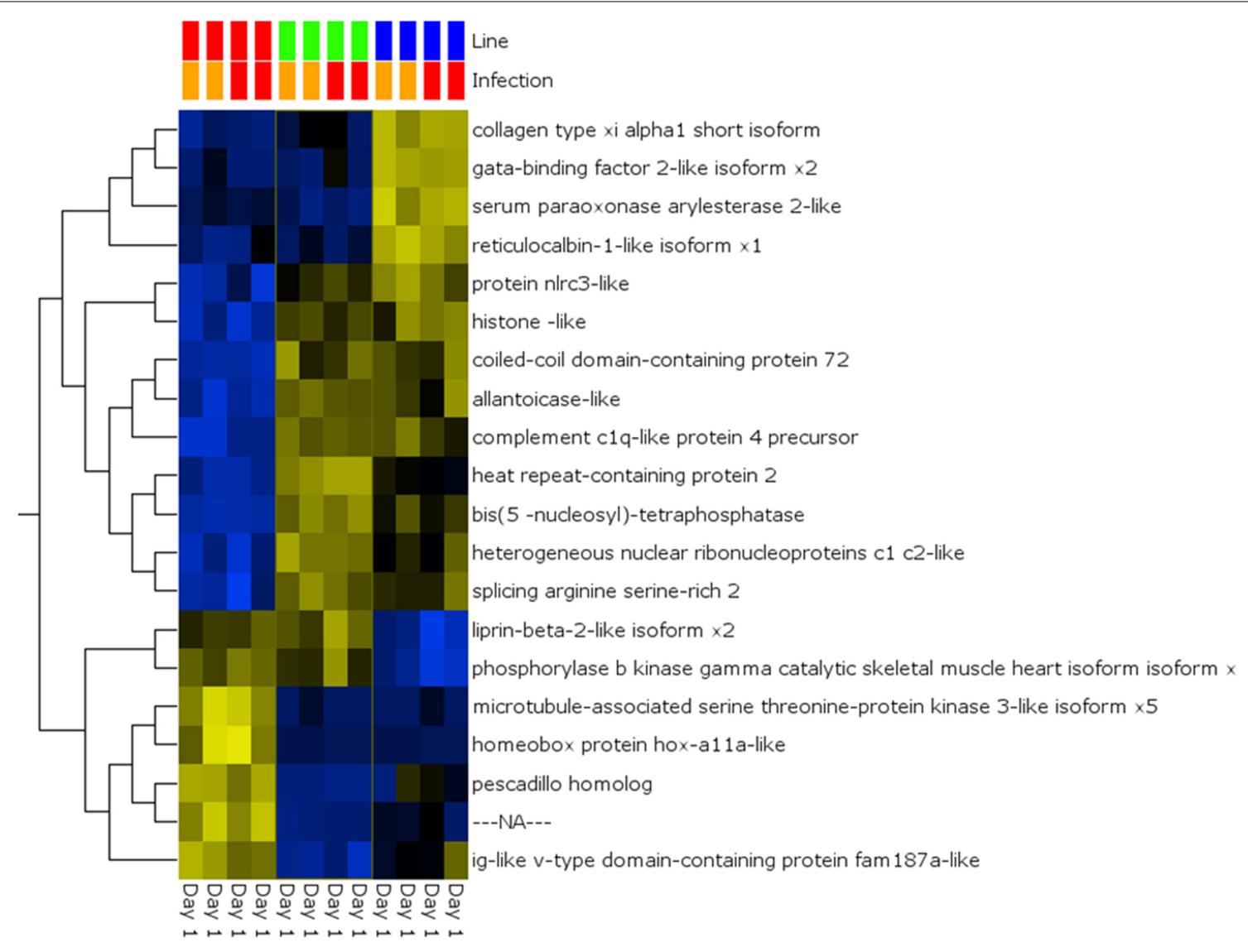

FIGURE 6 | Heat map showing multi-group comparison by genetic line eliminating infection as a factor $(q=0.05)$ for Day 1 samples. Variables (genes) are grouped by hierarchal clustering.

Table 4 | Fold-change in transcript abundance of genes described as modulated by Fp challenge.

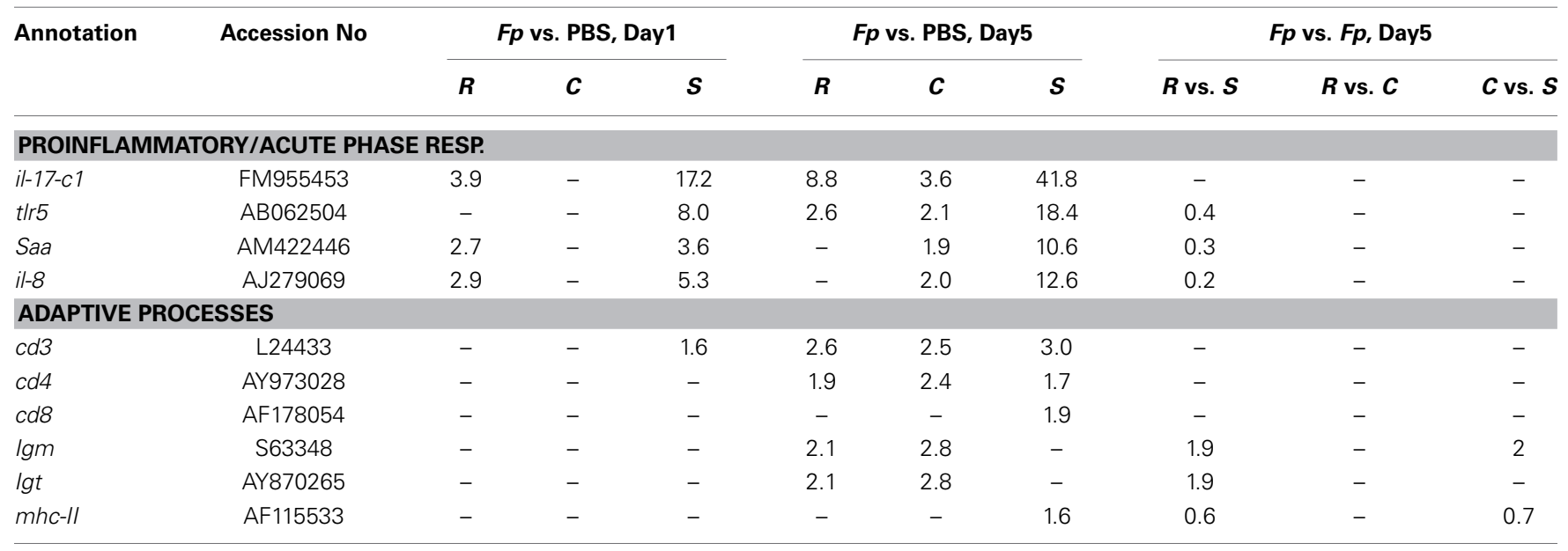

Quantification was performed between Fp and PBS challenged ARS-Fp-R (R), ARS-Fp-C (C), and ARS-Fp-S (S) line fish and between genetic lines on days 1 and 5 post-infection. There were no significant differences between genetic lines on day 1 post-infection.

\section{IDENTIFICATION OF LARGE PARALOGOUS FAMILIES OF PUTATIVE IMMUNE-RELEVANT GENES}

The recent rainbow trout genome contains expansion of putative gene families including protein nlrc3 and nlrc3-like $(n=111)$, protein nlrc5 $(n=9)$, polymeric immunoglobulin receptorlike $(n=14)$, perforin-1-like $(n=8)$, B-cell receptor cd22-like proteins $(n=38)$, cd209-like $(n=34)$, macrophage mannose receptor 1-like $(n=28)$, lrr and pyd domain-containing proteins 


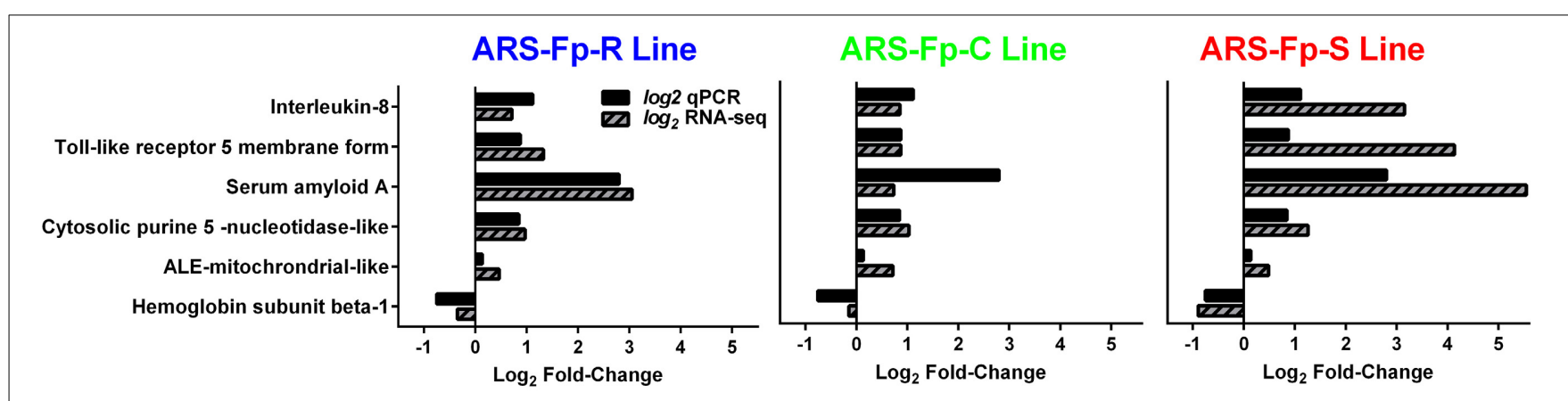

FIGURE 7 | Comparison between qPCR and RNA-seq by genetic line. All samples were from day 5 post-challenge.

$(n=37)$, and fish virus induced trim proteins $(n=44)$ (Supplementary Data Sheet 3 ). The availability of the genome allows a more comprehensive analysis of partially characterized immune gene families including tumor necrosis factor superfamily of ligands $(n=26)$ and toll-like receptors $(n=27)$ (Palti, 2011; Wiens and Glenney, 2011). Of note, a large number of tumor necrosis factor receptor superfamily members (tnfrsf, $n=59$ ) were identified (Supplementary Data Sheet 3). While the precise phylogenetic nomenclature and grouping of tnfrsf remains, our analysis indicates many members are differentially regulated in response to $F$. $p s y$ chrophilum infection including tnfrsf 1a precursor (GSONMT 00019008001), tnfrsf la-like (GSONMT00061996001) tnfrsf 5 precursor (GSONMT00012579001, GSONMT00003531001), tnfrsf 5-like (GSONMT00013182001), tnfrsf 6 (GSONMT 00082555001), tnfrsf 6b (GSONMT00034343001), tnfrsf 6blike (GSONMT00020936001), tnfrsf 9 (GSONMT00057532001), tnfrsf 9-like (GSONMT00050654001), tnfrsf 19-like isoform $\mathrm{x} 1$ (GSONMT00055755001) and tnfrsf 19-like isoform x2 (GSONMT00069336001). In addition, basal expression of one paralogue of tnfrsf 14-like isoform x1 (GSONMT00000915001, from a total $n=11$ paralogues present in the genome) is modestly higher in the ARS-Fp-R line as compared to the ARS-Fp-S line (Figure 5). While Blast2GO v.2 provides a description for novel sequences based on natural language text mining functionality (Gotz et al., 2008), we emphasize that detailed annotation and phylogenic analysis of these genes remains to be undertaken. Some of these genes may be pseudogenes and it is likely that the sequence description of many of the automated annotations we present here will require revision following expert curation, and may also change as improvements are made to the reference rainbow trout genome sequence and analysis of transcript variants.

\section{DIFFERENTIALLY EXPRESSED GENES BETWEEN NAIVE FISH FROM THE THREE GENETIC LINES}

There was tight clustering of data and a low number of gene expression differences between PBS-injected fish. This suggests phenotypic differences between the ARS-Fp-R, $-\mathrm{C}$, and -S lines are largely induced by infection and that selective breeding appears to have had a relatively low impact on basal gene expression during the normal physiologic state. Expression of complement factor-h like was observed to be significantly different between both naive and infected resistant and susceptible fish. Complement factor-h is a regulatory protein of the alternative complement pathway and although isolated from rainbow trout (Anastasiou et al., 2011), its contribution to the rainbow trout immune response has not yet been characterized.

\section{COMPARISON OF DIFFERENTIALLY REGULATED GENES WITH Langevin et al. STUDY}

This study expands on the work of Langevin et al. (2012) who described differential regulation of select genes by microarray and QPCR in the anterior kidney of BCWD resistant and susceptible rainbow trout clones after experimental infection. Both studies showed an increase in transcription of genes encoding pro-inflammatory cytokines, anti-bacterial effectors and matrix metalloproteases. Notably, complement factors, serum amyloid A, mannose-binding proteins, chemokines, and interleukins 1 and 8 all showed significant transcriptional increase on day 5 post-infection. RNA-seq data provides further evidence for upregulation of immunorelevant factors not previously identified, including tlr5, leptin, haptoglobin, and C-type lectin and genes associated with adaptive, physiologic, structural, and intracellular process. There was no evidence to support differential regulation of interferon-gamma during infection in either study although potential interferon induced genes are differentially regulated. Tumor necrosis factor-alpha was also notably absent from both studies despite previously published (Evenhuis and Cleveland, 2012) and unpublished data (Wiens, unpublished data) suggesting upregulation during infection. Assay sensitivity may have been confounded by whole-body RNA isolation which could reduce the ability to detect low abundant cytokines expressed in specific tissues.

There was variability between studies when transcriptomic responses were compared between resistant and susceptible line fish. Both studies observed higher bacterial loads in susceptible fish and increased transcriptional response of a number of genes associated with pro-inflammatory conditions including interleukin-1, cc chemokine and matrix metalloproteinases 1 and 19. Our study further identified serum amyloid A, differentially regulated trout protein, and cytochrome p450 la as significantly upregulated in susceptible fish. A number of metalloproteinase inhibitors were identified by Langevin et al. (2012) as significantly 
upregulated in resistant but not susceptible fish but showed no significant differences between genetic lines when analyzed in our study. This variability likely extends from apparent differences in experimental design including challenge route, bacterial dose and strain, tissue sampled, and microarray vs transcript count quantification and analysis. However, even with these differences in method, both studies demonstrated a robust immune response in F. psychrophilum challenged fish with significant differences in the transcriptome of resistant and susceptible fish, associated with immune relevant genes.

\section{CONCLUDING REMARKS}

Complex transcriptional differences were identified between lines following infection with F. psychrophilum strain CSF259-93. Most of the differentially regulated genes exhibited increased transcript abundance and correlated with higher bacterial loads. It is likely that most of the changes we identified are consequences of the differential expansion of $F$. psychrophilum following infection, especially in the ARS-Fp-S line. However, differences in bacterial load cannot account for transcriptional differences observed on day 1, as bacterial loads were similar between genetic lines. Future efforts will be directed at dissecting early time points following exposure and will focus on identifying inter-individual differences as this study examined pools of fish due to sequencing cost limitations. This data will be integrated with proteomic studies to examine the relationship between transcript and protein levels and to assist in exploring biomarkers of infection, immunocompetency and disease resistance. Also, in this study we did not analyze long non-coding RNA, microRNA and splice variants. Despite these limitations, we suggest that this data set represents an important future resource for exploration of candidate genes identified from QTL analyses being conducted in parallel with this study. Quantitative trait loci (QTL) mapping has identified nine QTL on seven chromosomes that have moderate to large effects on resistance (Vallejo et al., 2014a). We have focused on an Omy19 QTL (Wiens et al., 2013b) and confirmed inheritance in a subsequent generation (Vallejo et al., 2014b). By combining chromosomal position known for many of the genes within this dataset, we have begun to identify differentially regulated genes present on Omy19 and other chromosomes as proof of principal for this approach. Further studies are needed to validate and fine-map the BCWD QTL and these studies are currently underway. These complex patterns support a polygenetic architecture of BCWD resistance and will serve as a reference dataset for identifying mechanisms associated with the genetic basis of disease resistance.

\section{ACKNOWLEDGMENTS}

We acknowledge technical contributions from Travis Moreland and Joel Caren. Author contributions: conceived and designed the experiments: Yniv Palti, Mohamed Salem, Jianbo Yao, Gregory D. Wiens; performed the experiments Gregory D. Wiens, Bam Paneru, Hao Ma, Mohamed Salem, Alvaro G. Hernandez; analyzed the data David Marancik, Guangtu Gao, Gregory D. Wiens; contributed reagents/materials/analysis tools GG; wrote the paper David Marancik, Gregory D. Wiens. All authors reviewed and approved the manuscript. This work was supported by Agricultural Research Service CRIS Project 1930-32000-005 "Integrated Approaches for Improving Aquatic Animal Health in Cool and Cold Water Aquaculture," CRIS Project 1930-32000009 "Utilization of Genomics for Improving Production Traits in Cool and Cold Water Aquaculture" and Agriculture and Food Research Initiative competitive grant no. 2012-67015-30217 from the USDA National Institute of Food and Agriculture to Gregory D. Wiens. The authors have no conflict of interest to declare. Mention of trade names or commercial products in this publication is solely for the purpose of providing specific information and does not imply recommendation or endorsement by the U.S Department of Agriculture. USDA is an equal opportunity employer.

\section{SUPPLEMENTARY MATERIAL}

The Supplementary Material for this article can be found online at: http://www.frontiersin.org/journal/10.3389/fgene. 2014.00453/abstract

\section{REFERENCES}

Anastasiou, V., Mikrou, A., Papanastasiou, A. D., and Zarkadis, I. K. (2011). The molecular identification of factor $\mathrm{H}$ and factor I molecules in rainbow trout provides insights into complement C3 regulation. Fish Shellfish Immunol. 31, 491-499. doi: 10.1016/j.fsi.2011.06.008

Barnes, M. E., and Brown, M. L. (2011). A review of Flavobacterium psychrophilum biology, clinical signs, and bacterial cold water disease prevention and treatment. Open Fish Sci. J. 4, 40-48. doi: 10.2174/1874401X01104010040

Bayne, C. J., Gerwick, L., Fujiki, K., Nakao, M., and Yano, T. (2001). Immunerelevant (including acute phase) genes identified in the livers of rainbow trout, Oncorhynchus mykiss, by means of suppression subtractive hybridization. Dev. Comp. Immunol. 25, 205-217. doi: 10.1016/S0145-305X(00)00057-4

Beck, B. H., Farmer, B. D., Straus, D. L., Li, C., and Peatman, E. (2012). Putative roles for a rhamnose binding lectin in Flavobacterium columnare pathogenesis in channel catfish Ictalurus punctatus. Fish Shellfish Immunol. 33, 1008-1015. doi: 10.1016/j.fsi.2012.08.018

Benjamini, Y., and Hochberg, Y. (1995). Controlling the false discovery rate: a practical approach to multiple testing. J. R. Stat. Soc. B 57, 289-300.

Berthelot, C., Brunet, F., Chalopin, D., Juanchich, A., Bernard, M., Noel, B., et al. (2014). The rainbow trout genome provides novel insights into evolution after whole-genome duplication in vertebrates. Nat. Commun. 5, 3657. doi: 10.1038/ncomms4657

Bluthgen, N., Brand, K., Cajavec, B., Swat, M., Herzel, H., and Beule, D. (2005a). Biological profiling of gene groups utilizing Gene Ontology. Genome Inform. 16, 106-115. doi: 10.11234/gi1990.16.106

Bluthgen, N., Kielbasa, S. M., and Herzel, H. (2005b). Inferring combinatorial regulation of transcription in silico. Nucleic Acids Res. 33, 272-279. doi: $10.1093 /$ nar/gki167

Branson, E. (1995). Rainbow trout fry syndrome. Fish Vet. J. 1, 1-7.

Carrington, A. C., Collet, B., Holland, J. W., and Secombes, C. J. (2004). CpG oligodeoxynucleotides stimulate immune cell proliferation but not specific antibody production in rainbow trout (Oncorhynchus mykiss). Vet. Immunol. Immunopathol. 101, 211-222. doi: 10.1016/j.vetimm.2004.04.022

Cock, J., Gitterle, T., Salazar, M., and Rye, M. (2009). Breeding for disease resistance of Penaeid shrimps. Aquaculture 286, 1-11. doi: 10.1016/j.aquaculture.2008.09.011

Decostere, A., Lammens, M., and Haesebrouck, F. (2000). Difficulties in experimental infection studies with Flavobacterium psychrophilum in rainbow trout (Oncorhynchus mykiss) using immersion, oral and anal challenges. Res. Vet. Sci. 69, 165-169. doi: 10.1053/rvsc.2000.0408

Evenhuis, J. P., and Cleveland, B. M. (2012). Modulation of rainbow trout (Oncorhynchus mykiss) intestinal immune gene expression following bacterial challenge. Vet. Immunol. Immunopathol. 146, 8-17. doi: 10.1016/j.vetimm.2012.01.008

Gjedrem, T. (1983). Genetic variation in quantitative traits and selective breeding in fish and shellfish. Aquaculture 33, 51-71. doi: 10.1016/0044-8486(83)90386-1 
Gjedrem, T. (2005). Selection and Breeding Programs in Aquaculture. Dordrecht: Springer. doi: 10.1007/1-4020-3342-7

Gjedrem, T., Robinson, N., and Rye, M. (2012). The importance of selective breeding in aquaculture to meet future demands for animal protein: a review. Aquaculture 350-353, 117-129. doi: 10.1016/j.aquaculture.2012.04.008

Gómez, E., Méndez, J., Cascales, D., and Guijarro, J. A. (2014). Flavobacterium psychrophilum vaccine development: a difficult task. Microb. Biotech. 7, 414-423. doi: 10.1111/1751-7915.12099

Gotz, S., Garcia-Gomez, J. M., Terol, J., Williams, T. D., Nagaraj, S. H., Nueda, M. J., et al. (2008). High-throughput functional annotation and data mining with the Blast2GO suite. Nucleic Acids Res. 36, 3420-3435. doi: 10.1093/nar/gkn176

Hadidi, S., Glenney, G. W., Welch, T. J., Silverstein, J. T., and Wiens, G. D. (2008). Spleen size predicts resistance of rainbow trout to Flavobacterium psychrophilum challenge. J. Immunol. 180, 4156-4165. doi: 10.4049/jimmunol.180. 6.4156

Henriksen, M. M., Kania, P. W., Buchmann, K., and Dalsgaard, I. (2014). Effect of hydrogen peroxide and/or Flavobacterium psychrophilum on the gills of rainbow trout, Oncorhynchus mykiss (Walbaum). J. Fish Dis. doi: 10.1111/jfd.12232. [Epub ahead of print].

Jorgensen, J. B., Zou, J., Johansen, A., and Secombes, C. J. (2001). Immunostimulatory $\mathrm{CpG}$ oligodeoxynucleotides stimulate expression of IL-1beta and interferon-like cytokines in rainbow trout macrophages via a chloroquine-sensitive mechanism. Fish Shellfish Immunol. 11, 673-682. doi: 10.1006/fsim.2001.0344

Kent, M. L., Groff, J. M., Morrison, J. K., Yasutake, W. T., and Holt, R. A. (1989). Spiral swimming behavior due to cranial and vertebral lesions associated with Cytophaga psychrophila infections in salmonid fishes. Dis. Aquat. Org. 6, 11-16. doi: 10.3354/dao006011

Langevin, C., Blanco, M., Martin, S. A., Jouneau, L., Bernardet, J. F., Houel, A., et al. (2012). Transcriptional responses of resistant and susceptible fish clones to the bacterial pathogen Flavobacterium psychrophilum. PLoS ONE 7:e39126. doi: 10.1371/journal.pone.0039126

Langmead, B., Trapnell, C., Pop, M., and Salzberg, S. L. (2009). Ultrafast and memory-efficient alignment of short DNA sequences to the human genome. Genome Biol. 10, R25. doi: 10.1186/gb-2009-10-3-r25

Leeds, T. D., Silverstein, J. T., Weber, G. M., Vallejo, R. L., Palti, Y., Rexroad, C. E. III., et al. (2010). Response to selection for bacterial cold water disease resistance in rainbow trout. J. Anim. Sci. 88, 1936-1946. doi: 10.2527/jas.2009-2538

Lin, H. J., Shaffer, K. M., Sun, Z., Jay, G., He, W. W., and Ma, W. (2005). Glialderived nexin, a differentially expressed gene during neuronal differentiation, transforms HEK cells into neuron-like cells. Int. J. Dev. Neurosci. 23, 9-14. doi: 10.1016/j.ijdevneu.2004.09.007

Love, M. I., Huber, W., and Anders, S. (2014). Moderated estimation of fold change and dispersion for RNA-Seq data with DESeq2. bioRxiv 15:550. doi: 10.1101/002832

Madsen, L., Arnbjerg, J., and Dalsgaard, I. (2001). Radiological examination of the spinal column is farmed rainbow trout Oncorhynchus mykiss (Walbaum): experiments with Flavobacterium psychrophilum and oxytetracycline. Aquaculut. Res. 32, 235-241. doi: 10.1046/j.1365-2109.2001.00552.x

Marancik, D. P., Camus, M. S., Camus, A. C., Leeds, T. D., Weber, G. M., and Wiens, G. D. (2014a). Biochemical reference intervals and pathophysiological changes in Flavobacterium psychrophilum-resistant and -susceptible rainbow trout lines. Dis. Aquat. Org. 111, 239-248. doi: 10.3354/dao02777

Marancik, D. P., Leeds, T. D., and Wiens, G. D. (2014b). Histopathologic changes in disease-resistant-line and disease-susceptible-line juvenile rainbow trout experimentally infected with Flavobacterium psychrophilum. J. Aquat. Anim. Health 26, 181-189. doi: 10.1080/08997659.2014.920735

Marancik, D. P., and Wiens, G. D. (2013). A real-time polymerase chain reaction assay for identification and quantification of Flavobacterium psychrophilum and application to disease resistance studies in selectively bred rainbow trout Oncorhynchus mykiss. FEMS Microbiol. Lett. 339, 122-129. doi: 10.1111/15746968.12061

Martin, S. A., Blaney, S. C., Houlihan, D. F., and Secombes, C. J. (2006). Transcriptome response following administration of a live bacterial vaccine in Atlantic salmon (Salmo salar). Mol. Immunol. 43, 1900-1911. doi: 10.1016/j.molimm.2005.10.007

Mortazavi, A., Williams, B. A., McCue, K., Schaeffer, L., and Wold, B. (2008). Mapping and quantifying mammalian transcriptomes by RNA-Seq. Nat. Methods 5, 621-628. doi: 10.1038/nmeth.1226
Nematollahi, A., Decostere, A., Pasmans, F., and Haesebrouck, F. (2003). Flavobacterium psychrophilum infections in salmonid fish. J. Fish Dis. 26, 563-574. doi: 10.1046/j.1365-2761.2003.00488.x

Nilsen, H., Olsen, A. B., Vaagnes, O., Hellberg, H., Bottolfsen, K., Skjelstad, H., et al. (2011). Systemic Flavobacterium psychrophilum infection in rainbow trout, Oncorhynchus mykiss (Walbaum), farmed in fresh and brackish water in Norway. J. Fish Dis. 34, 403-408. doi: 10.1111/j.1365-2761.2011.01249.x

Ojha, N., Roy, S., He, G., Biswas, S., Velayutham, M., Khanna, S., et al. (2008). Assessment of wound-site redox environment and the significance of Rac2 in cutaneous healing. Free Radic. Biol. Med. 44, 682-691. doi: 10.1016/j.freeradbiomed.2007.10.056

Orieux, N., Douet, D. G., Le Henaff, M., and Bourdineaud, J. P. (2013). Prevalence of Flavobacterium psychrophilum bacterial cells in farmed rainbow trout: characterization of metallothionein A and interleukin1-beta genes as markers overexpressed in spleen and kidney of diseased fish. Vet. Microbiol. 162, 127-135. doi: 10.1016/j.vetmic.2012.08.015

Overturf, K., and LaPatra, S. (2006). Quantitative expression (Walbaum) of immunological factors in rainbow trout, Oncorhynchus mykiss (Walbaum), after infection with either Flavobacterium psychrophilum, Aeromonas salmonicida, or infectious haematopoietic necrosis virus. J. Fish Dis. 29, 215-224. doi: 10.1111/j.1365-2761.2006.00707.x

Palti, Y. (2011). Toll-like receptors in bony fish: from genomics to function. Dev. Comp. Immunol. 35, 1263-1272. doi: 10.1016/j.dci.2011.03.006

Peatman, E., Li, C., Peterson, B. C., Straus, D. L., Farmer, B. D., and Beck, B. H. (2013). Basal polarization of the mucosal compartment in Flavobacterium columnare susceptible and resistant channel catfish (Ictalurus punctatus). Mol. Immunol. 56, 317-327. doi: 10.1016/j.molimm.2013.04.014

Pereiro, P., Dios, S., Boltaña, S., Coll, J., Estepa, A., Mackenzie, S., et al. (2014). Transcriptome profiles associated to VHSV infection or DNA vaccination in turbot (Scophthalmus maximus). PLoS ONE 9:e104509. doi: 10.1371/journal.pone.0104509

Romer, J., Bugge, T. H., Pyke, C., Lund, L. R., Flick, M. J., Degen, J. L., et al. (1996). Impaired wound healing in mice with a disrupted plasminogen gene. Nat. Med. 2, 287-292. doi: 10.1038/nm0396-287

Schmittgen, T. D., and Livak, K. J. (2008). Analyzing real-time PCR data by the comparative $\mathrm{C}(\mathrm{T})$ method. Nat. Protoc. 3, 1101-1108. doi: 10.1038/nprot.2008.73

Shi, M., Huang, R., Du, F., Pei, Y., Liao, L., Zhu, Z., et al. (2014). RNA-seq profiles from grass carp tissues after reovirus (GCRV) infection based on singular and modular enrichment analyses. Mol. Immunol. 61, 44-53. doi: 10.1016/j.molimm.2014.05.004

Silverstein, J. T., Vallejo, R. L., Palti, Y., Leeds, T. D., Rexroad, C. E. III., Welch, T. J., et al. (2009). Rainbow trout resistance to bacterial cold-water disease is moderately heritable and is not adversely correlated with growth. J. Anim. Sci. 87, 860-867. doi: 10.2527/jas.2008-1157

Stadnyk, A. W. (1994). Cytokine production by epithelial cells. FASEB J. 8, 1041-1047.

Sundell, K., Högfors-Rönnholm, E., and Wiklund, T. (2014). "23 vaccination against diseases caused by flavobacteriaceae species," in Fish Vaccination, eds A. L. Roar Gudding and O. Evensen (Chichester: Wiley Blackwell), 273-282. doi: 10.1002/9781118806913.ch23

Sunyer, J. O., and Lambris, J. D. (1998). Evolution and diversity of the complement system of poikilothermic vertebrates. Immunol. Rev. 166, 39-57. doi: 10.1111/j.1600-065X.1998.tb01251.x

Tsoi, S. C., Ewart, K. V., Penny, S., Melville, K., Liebscher, R. S., Brown, L. L., et al. (2004). Identification of immune-relevant genes from atlantic salmon using suppression subtractive hybridization. Mar. Biotechnol. 6, 199-214. doi: 10.1007/s10126-002-0101-2

Vallejo, R. L., Palti, Y., Liu, S., Evenhuis, J. P., Gao, G., Rexroad, C. E. III., et al. (2014a). Detection of QTL in rainbow trout affecting survival when challenged with Flavobacterium psychrophilum. Mar. Biotechnol. 16, 349-360. doi: 10.1007/s10126-013-9553-9

Vallejo, R. L., Palti, Y., Liu, S., Marancik, D. P., and Wiens, G. D. (2014b). Validation of linked QTL for bacterial cold water disease resistance and spleen size on rainbow trout chromosome Omy19. Aquaculture 432, 139-143. doi: 10.1016/j.aquaculture.2014.05.003

Van Muiswinkel, W. B., Wiegertjes, G. F., and Stet, R. J. M. (1999). The influence of environmental and genetic factors on the disease resistance of fish. Aquaculture 172, 103-110. doi: 10.1016/S0044-8486(98)00444-X 
Villarroel, F., Casado, A., Vasquez, J., Matamala, E., Araneda, B., Amthauer, R., et al. (2008). Serum amyloid A: a typical acute-phase reactant in rainbow trout? Dev. Comp. Immunol. 32, 1160-1169. doi: 10.1016/j.dci.2008.03.004

Villarroel, F., Zambrano, A., Amthauer, R., and Concha, M. I. (2009). Detection of up-regulated serum amyloid A transcript and of amyloid AA aggregates in skeletal muscle lesions of rainbow trout infected with Flavobacterium psychrophilum. Vet. Immunol. Immunopathol. 130, 120-124. doi: 10.1016/j.vetimm.2009. 02.002

Wang, T., Martin, S. A., and Secombes, C. J. (2009). Two interleukin-17C-like genes exist in rainbow trout Oncorhynchus mykiss that are differentially expressed and modulated. Dev. Comp. Immunol. 34, 491-500. doi: 10.1016/j.dci.2009.11.011

Whyte, S. K. (2007). The innate immune response of finfish-a review of current knowledge. Fish Shellfish Immunol. 23, 1127-1151. doi: 10.1016/j.fsi.2007.06.005

Wiens, G. D., and Glenney, G. W. (2011). Origin and evolution of TNF and TNF receptor superfamilies. Dev. Comp. Immunol. 35, 1324-1335. doi: 10.1016/j.dci.2011.03.031

Wiens, G. D., LaPatra, S. E., Welch, T. J., Evenhuis, J. P., Rexroad, C. E. III., and Leeds, T. D. (2013a). On-farm performance of rainbow trout (Oncorhynchus mykiss) selectively bred for resistance to bacterial cold water disease: effect of rearing environment on survival phenotype. Aquaculture 388-391, 128-136. doi: 10.1016/j.aquaculture.2013.01.018

Wiens, G. D., LaPatra, S. E., Welch, T. J., Rexroad, C. E. III., Call, D. R., Cain, K. D., et al. (2014). Complete genome sequence of Flavobacterium psychrophilum strain CSF259-93 used select rainbow trout for increased genetic resistance against bacterial cold water disease. Genome Announcements 2, e00889-14. doi: 10.1128/genomeA.00889-14
Wiens, G. D., Vallejo, R. L., Leeds, T. D., Palti, Y., Hadidi, S., Liu, S., et al. (2013b). Assessment of genetic correlation between bacterial cold water disease resistance and spleen index in a domesticated population of rainbow trout: identification of QTL on chromosome Omy19. PLoS ONE 8:e75749. doi: 10.1371/journal.pone.0075749

Woods, A., and Couchman, J. R. (1998). Syndecans: synergistic activators of cell adhesion. Trends Cell Biol. 8, 189-192. doi: 10.1016/S0962-8924(98)01244-6

Conflict of Interest Statement: The authors declare that the research was conducted in the absence of any commercial or financial relationships that could be construed as a potential conflict of interest.

Received: 24 October 2014; paper pending published: 24 November 2014; accepted: 11 December 2014; published online: 08 January 2015.

Citation: Marancik D, Gao G, Paneru B, Ma H, Hernandez AG, Salem M, Yao J, Palti $Y$ and Wiens GD (2015) Whole-body transcriptome of selectively bred, resistant-, control-, and susceptible-line rainbow trout following experimental challenge with Flavobacterium psychrophilum. Front. Genet. 5:453. doi: 10.3389/fgene.2014.00453 This article was submitted to Livestock Genomics, a section of the journal Frontiers in Genetics.

Copyright (C) 2015 Marancik, Gao, Paneru, Ma, Hernandez, Salem, Yao, Palti and Wiens. This is an open-access article distributed under the terms of the Creative Commons Attribution License (CC BY). The use, distribution or reproduction in other forums is permitted, provided the original author(s) or licensor are credited and that the original publication in this journal is cited, in accordance with accepted academic practice. No use, distribution or reproduction is permitted which does not comply with these terms. 